\title{
K2-114b and K2-115b: Two Transiting Warm Jupiters
}

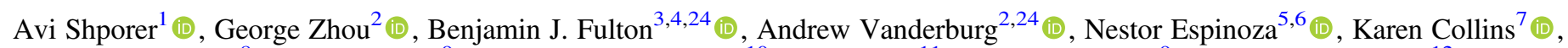
David Ciardi $^{8}$, Daniel Bayliss 9 (10), James D. Armstrong ${ }^{10}$, Joao Bento ${ }^{11}$, Francois Bouchy ${ }^{9}$, William D. Cochran ${ }^{12}$ (1), Andrew Collier Cameron ${ }^{13}$, Knicole Colón ${ }^{14}$ (i), Ian Crossfield ${ }^{15}$, Diana Dragomir ${ }^{16,25}$ (D) , Andrew W. Howard ${ }^{4}$,

Steve B. Howell ${ }^{17}$ (D) , Howard Isaacson ${ }^{18}$ (D) , John F. Kielkopf ${ }^{19}$, Felipe Murgas ${ }^{20,21}$, Ramotholo Sefako ${ }^{22}$, Evan Sinukoff ${ }^{3,4} \mathbb{D}_{\mathbb{D}}$, Robert Siverd $^{23}$ (D) , and Stephane Udry ${ }^{9}$

${ }^{1}$ Division of Geological and Planetary Sciences, California Institute of Technology, Pasadena, CA 91125, USA

${ }^{2}$ Harvard-Smithsonian Center for Astrophysics, 60 Garden Street, Cambridge, MA 02138, USA

${ }^{3}$ Institute for Astronomy, University of Hawaii, Honolulu, HI 96822, USA

${ }^{4}$ California Institute of Technology, Pasadena, CA, USA

${ }^{5}$ Instituto de Astrofísica, Facultad de Física, Pontificia Universidad Católica de Chile, Av. Vicuña Mackenna 4860, 782-0436 Macul, Santiago, Chile

${ }^{6}$ Millennium Institute of Astrophysics, Av. Vicuña Mackenna 4860, 782-0436 Macul, Santiago, Chile

${ }^{7}$ Department of Physics \& Astronomy, Vanderbilt University, Nashville, TN 37235, USA

${ }^{8}$ NASA Exoplanet Science Institute/Caltech, Pasadena, CA, USA

${ }_{9}^{9}$ Observatoire Astronomique de l'Universit'e de Geneve, $51 \mathrm{ch}$. des Maillettes, 1290 Versoix, Switzerland

${ }^{10}$ University of Hawaii Institute for Astronomy 34 Ohia Ku Street, Pukalani, HI 96768, USA

${ }^{11}$ Research School of Astronomy and Astrophysics, Mount Stromlo Observatory, Australian National University, Weston, ACT 2611, Australia

${ }^{12}$ McDonald Observatory and Department of Astronomy, University of Texas, Austin, TX 78812, USA

${ }^{13}$ Centre for Exoplanet Science, SUPA School of Physics \& Astronomy, University of St Andrews, North Haugh, St Andrews KY16 9SS, UK

${ }^{15}$ NASA Goddard Space Flight Center, Greenbelt, MD 20771, USA

${ }_{16}$ Astronomy and Astrophysics Department, UC Santa Cruz, CA, USA

${ }^{16}$ Massachusetts Institute of Technology, Cambridge, MA 02139 USA

${ }_{17}^{17}$ NASA Ames Research Center, Moffett Field, CA 94035, USA

${ }^{18}$ Astronomy Department, University of California, Berkeley, CA, USA

${ }^{19}$ Department of Physics \& Astronomy, University of Louisville, Louisville, KY 40292, USA

${ }^{20}$ Instituto de Astrofísica de Canarias, Vía Láctea s/n, E-38205 La Laguna, Tenerife, Spain

${ }^{21}$ Departamento de Astrofísica, Universidad de La Laguna, Spain

${ }^{22}$ South African Astronomical Observatory, P.O. Box 9, Observatory, 7935, South Africa

${ }^{23}$ Las Cumbres Observatory Global Telescope Network, 6740 Cortona Drive, Suite 102, Santa Barbara, CA 93117, USA

Received 2017 June 23; revised 2017 September 3; accepted 2017 September 6; published 2017 October 20

\begin{abstract}
We report the first results from a search for transiting warm Jupiter exoplanets_-gas giant planets receiving stellar irradiation below about $10^{8} \mathrm{erg} \mathrm{s}^{-1} \mathrm{~cm}^{-2}$, equivalent to orbital periods beyond about 10 days around Sun-like stars. We have discovered two transiting warm Jupiter exoplanets initially identified as transiting candidates in $K 2$ photometry. K2-114b has a mass of $1.85_{-0.22}^{+0.23} M_{\mathrm{J}}$, a radius of $0.942_{-0.020}^{+0.032} R_{\mathrm{J}}$, and an orbital period of 11.4 days. $\mathrm{K} 2-115 \mathrm{~b}$ has a mass of $0.84_{-0.20}^{+0.18} M_{\mathrm{J}}$, a radius of $1.115_{-0.061}^{+0.057} R_{\mathrm{J}}$, and an orbital period of 20.3 days. Both planets are among the longest-period transiting gas giant planets with a measured mass, and they are orbiting relatively old host stars. Both planets are not inflated, as their radii are consistent with theoretical expectations. Their position in the planet radius-stellar irradiation diagram is consistent with the scenario where the radius-irradiation correlation levels off below about $10^{8} \mathrm{erg} \mathrm{s}^{-1} \mathrm{~cm}^{-2}$, suggesting that for warm Jupiters stellar irradiation does not play a significant role in determining the planet radius. We also report our identification of another $K 2$ transiting warm Jupiter candidate, EPIC 212504617, as a false positive.
\end{abstract}

Key words: stars: individual (K2-114, EPIC 211418729, K2-115, EPIC 211442297, EPIC 212504617)

Supporting material: machine-readable table

\section{Introduction}

The number of known transiting hot Jupiter exoplanets-gas giant exoplanets in short orbital periods of only a few days-is now in the few hundreds. Despite that large number there are still several open questions related to this class of exoplanets. Many, if not most, of the known hot Jupiters have larger radii than theoretically expected (e.g., Weiss et al. 2013; Baraffe et al. 2014; Lopez \& Fortney 2016). Although various explanations have been proposed, none have completely solved this puzzle, suggesting there is more than a single mechanism at play here, and/or that we are missing some physics-shaping planetary structure (Baraffe

\footnotetext{
${ }^{24}$ National Science Foundation Graduate Research Fellow.

25 NASA Hubble Fellow.
}

et al. 2014). Another hot Jupiter mystery is their formation and orbital evolution. While several theories have been put forth (e.g., Lin et al. 1996; Rasio \& Ford 1996; Alibert et al. 2005; Fabrycky \& Tremaine 2007), it is still not clear how gas giant planets reach short orbits around Sun-like stars, at only a few 0.01 au.

One way to shed light on the above questions is to examine the population of warm Jupiters-gas giant planets receiving stellar irradiation below $10^{8} \mathrm{erg} \mathrm{s}^{-1} \mathrm{~cm}^{-2}$, corresponding to orbital periods beyond $\approx 10$ days around Sun-like stars. Specifically, we would like to examine their planet radii and orbital eccentricities. However, this is difficult, as there are currently only a handful of confirmed transiting warm Jupiters with measured orbits and masses.

We have initiated a ground-based follow-up campaign of warm Jupiter transiting candidates in order to increase the 
Table 1

Basic Targets Information

\begin{tabular}{lcc}
\hline \hline Parameter & K2-114 & K2-115 \\
\hline EPIC & 211418729 & 211442297 \\
R.A. & $08: 31: 31.911$ & $08: 26: 12.827$ \\
Decl. & $11: 55: 20.15$ & $12: 16: 54.97$ \\
$g$ (mag) & 15.07 & 13.59 \\
$K_{p}$ (mag) & 14.29 & 13.19 \\
$r$ (mag) & 14.24 & 13.19 \\
$i$ (mag) & 13.95 & 13.02 \\
$H$ (mag) & 12.84 & 12.11 \\
$K$ (mag) & 12.39 & 11.76 \\
\hline
\end{tabular}

number of known transiting warm Jupiters that are confirmed as planets, i.e., their masses and orbits are measured. This campaign is part of a Las Cumbres Observatory (LCO; Brown et al. 2013) Key Project ${ }^{26}$ (PI: Avi Shporer). Our current primary source of transiting candidates is the $K 2$ mission (Howell et al. 2014) and in the future the TESS mission (Ricker et al. 2015; Sullivan et al. 2015). We report here the confirmation of two $K 2$ warm Jupiters orbiting K2-114 and $\mathrm{K} 2-115$, which are the first discoveries from this project. Basic information about the two targets is given in Table 1 .

We give a more detailed motivation for our search for transiting warm Jupiter exoplanets in Section 2. In Section 3 we describe our observations of K2-114 and K2-115, in Section 4 we describe our data analysis and results, and we discuss our new discoveries in Section 5.

\section{Why Warm Jupiters?}

Our scientific goals are to (1) investigate the inflated gas giant conundrum, (2) study the mystery of hot Jupiter orbital evolution, and (3) identify targets for extending exoplanet atmosphere and stellar obliquity studies beyond the hot Jupiter class.

Briefly, the suggested mechanisms responsible for inflating gas giants can be divided into three categories (Weiss et al. 2013; Lopez \& Fortney 2016). (I) One mechanism is inflation due to stellar irradiation transported from the planet's atmosphere to its interior (e.g., Ginzburg \& Sari 2016; Komacek \& Youdin 2017) through, e.g., Ohmic dissipation (Batygin \& Stevenson 2010), thermal tides (Arras \& Socrates 2010), kinetic energy transport (Showman \& Guillot 2002), mechanical greenhouse (Youdin \& Mitchell 2010), or advection of potential energy (Tremblin et al. 2017). (II) Another mechanism is inflation due to tidal heating following orbital eccentricity dissipation (Bodenheimer et al. 2001). If the planet eccentricity is continuously being excited, for example, through interaction with a third body, this inflation mechanism can be long-lived. (III) A third mechanism is delayed contraction due to, e.g., increased atmospheric opacities (Burrows et al. 2007). Unlike the first two categories, the third category affects all giant planets, not only those on short or eccentric orbits.

One clue for understanding inflated gas giants is the empirical correlation between planet radius $R_{p}$ and stellar irradiation $f$ (referred to hereafter as the radius-irradiation correlation, or $R_{p}-f$ correlation; e.g., Fortney et al. 2007; Laughlin et al. 2011; Enoch et al. 2012; Lopez \& Fortney 2016). While it is consistent

\footnotetext{
${ }^{26}$ http://web.gps.caltech.edu/ shporer/LCOKP/
}

with inflation through irradiation, it does not identify which of the category I mechanisms listed above is the dominant one, and, we must keep in mind that correlation does not necessarily mean causation. The fact that the vast majority of known transiting gas giant exoplanets are at short periods, typically within 10 days or with irradiation above $10^{8} \mathrm{erg} \mathrm{s}^{-1} \mathrm{~cm}^{-2}$, hinders a detailed understanding of the $R_{p}-f$ correlation. While it seems that the correlation levels off at some irradiation level, the exact behavior is not clear, raising a few questions. How low in irradiation does this correlation stretch? Is the lack of inflated warm Jupiters a robust feature? What is the irradiation below which there are no inflated gas giants? What drives the scatter in the correlation?

Regarding hot Jupiter orbital evolutions (goal 2 above), several theories attempt to explain how gas giants reach short orbital periods. Some invoke interaction with another object in the system (another planet or a stellar binary companion) where the gas giant planet is first injected into an eccentric orbit which then undergoes tidal circularization (e.g., Rasio \& Ford 1996; Fabrycky \& Tremaine 2007). Other theories suggest processes where the gas giant planet moves to an inward orbit by interacting with the protoplanetary disk, during which the orbit is kept circular (e.g., Lin et al. 1996; Alibert et al. 2005). Therefore, the two types of scenarios above differ in the orbital eccentricity of the gas giant planet as it is migrating from a wide orbit to a short orbit. This means that the orbital eccentricity of warm Jupiters is a clue to identifying the dominant orbital evolution channel of hot Jupiters, and is another motivation for expanding the known warm Jupiter sample to support statistical analysis. Some evidence that a significant fraction of warm Jupiters have circular orbits was gathered by studying the occurrence of additional planets in systems containing warm Jupiters compared to systems containing hot Jupiters. The increased occurrence of short-period planet companions to warm Jupiters (e.g., Steffen et al. 2012; Dong et al. 2014; Huang et al. 2016) suggests that their orbits are circular since non-circular orbits are expected to make the multi-planet system dynamically unstable.

Another gap we wish to bridge with this program is the very small number of warm Jupiters available for exoplanet atmosphere and stellar obliquity studies (goal 3 above). For the bright stars in our sample detections of warm Jupiters will enable the study of exoplanet atmospheres with lower equilibrium temperatures, and the study of stellar obliquity in star-planet systems with weaker tidal interactions.

\section{Observations}

\subsection{K2 Photometry}

The two targets were initially identified as transiting planet candidates in $K 2$ Campaign 5 photometry. They were observed by $K 2$ in long-cadence (30-minute integration time) from 2015 April 27 to July 10 . We reduced the $K 2$ light curves following Vanderburg \& Johnson (2014) and Vanderburg et al. (2016). We then looked for transit signals using the Box-Least-Squares periodogram search (Kovács et al. 2002), as implemented by Vanderburg et al. (2016). Upon identifying transit candidates, we checked that they did not show known signs of a false positive by looking at the centroid motion of the target star during transit, searching for secondary eclipses, inspecting each individual transit, and confirming that the transit signal did not 

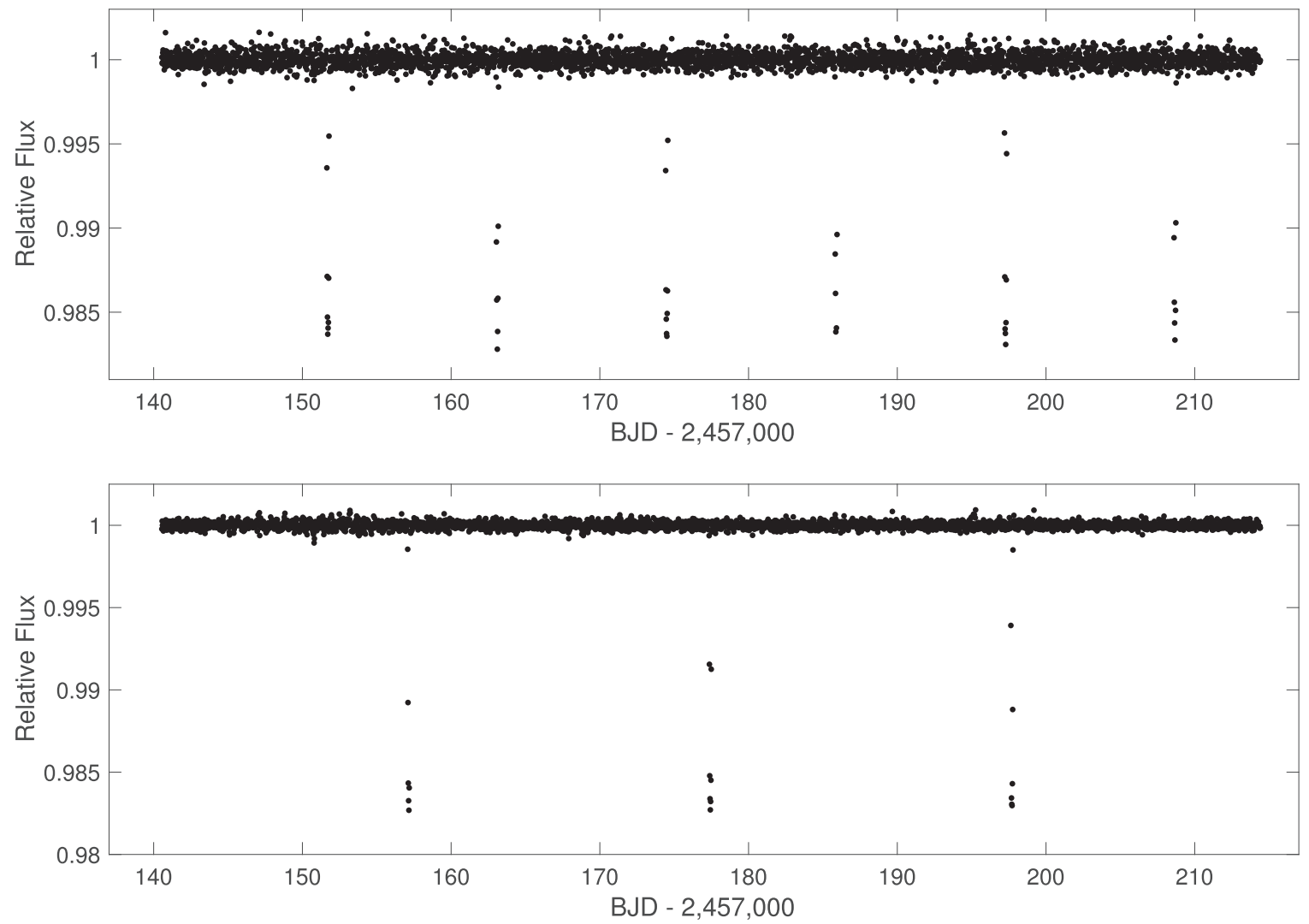

Figure 1. Complete, detrended, and normalized K2 Campaign 5 light curves of K2-114 (top), including 6 transit events, and of K2-115 (bottom), including 3 transit events.

change significantly in light curves extracted from different photometric apertures. We then re-processed the light curves by simultaneously fitting for the transits, $K 2$ thruster systematics, and low-frequency variations using the method described by Vanderburg et al. (2016). The complete, detrended, and normalized $K 2$ Campaign 5 light curves are shown in Figure 1 and the phase-folded transit light curves are shown in Figure 2. No additional transit signals were identified in the $K 2$ light curve of both targets, nor was a secondary eclipse signal detected.

We have searched for the host stars' rotation periods by calculating their $K 2$ light curve autocorrelation (McQuillan et al. 2014). We used the non-detrended $K 2$ light curves while masking out the in-transit data. These light curves are shown in Figure 3 along with the autocorrelation function. The periodicity detected for $\mathrm{K} 2-114$ is close to the $K 2$ time span where it is challenging to separate stellar variability and longterm systematic features in the $K 2$ data. Therefore, we do not claim a detection of the rotation period for that star. For K2-115 we identify a 22.2-day periodicity, visually identified in the non-detrended light curve, interpreted as the host star's rotation period. We estimate this rotation period uncertainty to be at the $10 \%$ level to account for differential rotation (e.g., Reinhold et al. 2013), since during the limited $K 2$ data time span the star spots are likely to be located within a narrow latitude range that is not known. The K2-115 host star rotation period is longer but close to the 20.3-day orbital period. Although given its mass and relatively long orbital period, the planet is not expected to tidally synchronize the star's rotation (e.g., Mazeh 2008).

\subsection{High Angular Resolution Imaging}

After identifying the transit candidates in $K 2$ photometry we checked that they are isolated targets using high angular resolution imaging. The imaging data were acquired as part of wider programs using Keck II and Gemini-North to obtain infrared adaptive optics (AO) and optical speckle imaging. The AO observations utilized the target stars as natural guide stars; the NIRC2 camera was utilized on Keck II and the NIRI camera was utilized on Gemini-North. NIRC2 has a pixel scale of 0 ."00942/pixel and NIRI, with the Altair AO system, has a pixel scale of 0 ". 0214/pixel (Hodapp et al. 2003). The GeminiNorth speckle observations were obtained with the visiting instrument DSSI, which has a pixel scale of 0 ". $011 /$ pixel (Horch et al. 2009, 2012).

For K2-114 the Keck AO data were obtained on 2016 February 19 with the Kp filter and an integration time of $17 \mathrm{~s}$ per frame for a total of $153 \mathrm{~s}$, and the Gemini-North AO data were obtained on 2016 February 20 with the K filter and an integration time of $5 \mathrm{~s}$ per frame for a total of $50 \mathrm{~s}$. For K2-115 the Keck AO data were obtained on 2016 January 21 with the $\mathrm{Br}$ gamma filter and an integration time of $30 \mathrm{~s}$ per frame for a total of $270 \mathrm{~s}$. The Keck AO data have resolutions of 0."06-0."07 (FWHM) and the Gemini AO data have a resolution of 0 ". 09 (FWHM).

The speckle data were obtained only for K2-115, with the 692 and $883 \mathrm{~nm}$ filters on 2016 January 13. The data were obtained with $100060 \mathrm{~ms}$ frames in both filters simultaneously. The speckle imaging is produced through a Fourier reconstruction of the speckle interferogram and have an angular resolution of 0. "02 (Horch et al. 2012). 

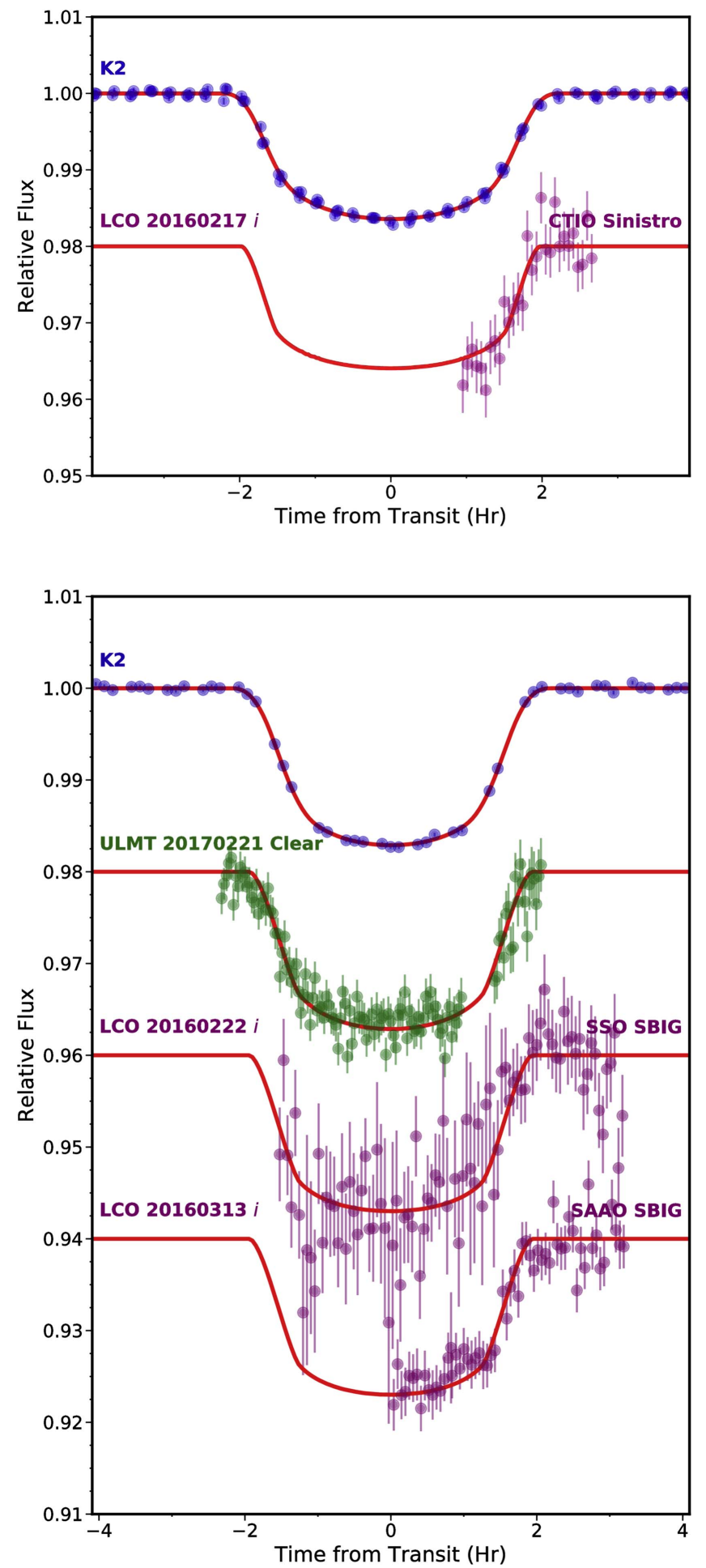

Figure 2. Transit light curves of K2-114b (top) and K2-115b (bottom). The $K 2$ light curves are phase-folded and plotted in blue, and ground-based follow-up light curves are plotted and labeled below and are arbitrarily offset in flux for visibility. The solid red line is the best-fit global model.

The sensitivity of the AO data was determined by injecting fake sources into the final combined images with separations from the primary targets in integer multiples of the central source's FWHM (Furlan et al. 2017). For the speckle data, the sensitivity was estimated from the scatter in the reconstructed image (Horch et al. 2011; Furlan et al. 2017). In both cases the sensitivity curves (contrast curves) represent $5 \sigma$ limits and are shown in Figure 4.

For both targets, no stellar companions were detected in either the infrared AO or the optical speckle imaging, indicating (to the limits of the data) that the stars appear to be single stars with no additional components to either dilute the transit depths or confuse the determination of the origin of the transit signal (e.g., Ciardi et al. 2015).

\subsection{Keck/HIRES Spectroscopy and Radial Velocity (RV) Monitoring}

Once we confirmed that the two targets appear isolated in high angular resolution imaging, we began gathering highresolution spectra with the HIRES instrument mounted on the Keck I telescope (Vogt et al. 1994).

We collected a total of six spectra of K2-114 between 2016 February 2 and 2017 May 13. The first measurement was collected without the iodine cell in the light path for spectral characterization and searching for secondary lines (Kolbl et al. 2015). The remaining five spectra were observed with the iodine cell in the light path, which imprints a dense forest of molecular absorption lines to be used as a simultaneous wavelength and instrumental point-spread function reference, in order to measure the target's RV. The signal-to-noise ratio $(\mathrm{S} / \mathrm{N})$ for each spectrum was $\approx 40$ per pixel and exposure times were typically 20 minutes. All spectra were collected using the 0 ". $86 \times 14^{\prime \prime}$ slit, for a resolution of about 65,000 .

We collected eight spectra and seven RV measurements using the iodine cell for K2-115 between 2016 February 4 and 2017 April 10. Our setup was identical to that for K2-114, except exposure times were generally shorter, $\approx 10$ minutes, and $\mathrm{S} / \mathrm{N}$ was slightly higher, $\approx 45$ per pixel.

RVs for both stars were extracted by forward-modeling the composite iodine+stellar spectra in $\approx 800$ small spectral chunks following the method of Butler \& Marcy (1996). We used the SpecMatch package (Petigura 2015) to synthesize an iodine-free stellar spectrum to be used in the modeling process (Fulton et al. 2015), instead of collecting an expensive, long, high $\mathrm{S} / \mathrm{N}$, iodine-free exposure of the target.

We also used SpecMatch to extract the spectroscopic stellar parameters from our single iodine-free observation of each star. Those include the effective temperature $T_{\text {eff }}$, surface gravity $\log g$, metallicity $[\mathrm{Fe} / \mathrm{H}]$, and stellar rotation projected on the line of sight $V \sin (I)$, where $V$ is the equatorial rotation and $I$ is the stellar rotation inclination angle. The SpecMatch results for the spectral parameters of both targets are listed in Table 2, and the RVs of both targets are listed in Table 3 and plotted in Figure 5.

In addition, we have calculated the activity indicators $S_{H K}$ and $\log R_{H K}^{\prime}$ (Isaacson \& Fischer 2010) for each of the Keck/ HIRES spectra, and list in Table 2 the mean of these indicators for the two host stars. For the indicators' uncertainties we adopt the scatter (standard deviation) in each sample. That scatter is affected primarily by the low $\mathrm{S} / \mathrm{N}$ of the spectra in the $\mathrm{Ca} \mathrm{H} \&$ $\mathrm{K}$ lines, which is 3-8 for $\mathrm{K} 2-114$ and 8-18 for $\mathrm{K} 2-115$. Although K2-114 activity indicators suggest it is more active than $\mathrm{K} 2-115$, we have detected a rotation period for the latter and not for the former (See Section 3.1). This could be because the rotation period is too long to be detected in $K 2$ data, consistent with the star being a slow rotator $\left(V \sin (I)<2 \mathrm{~km} \mathrm{~s}^{-1}\right)$. As seen in Figure 3 we have identified a possible periodicity for $\mathrm{K} 2-114$ of 65.2 days, but the 

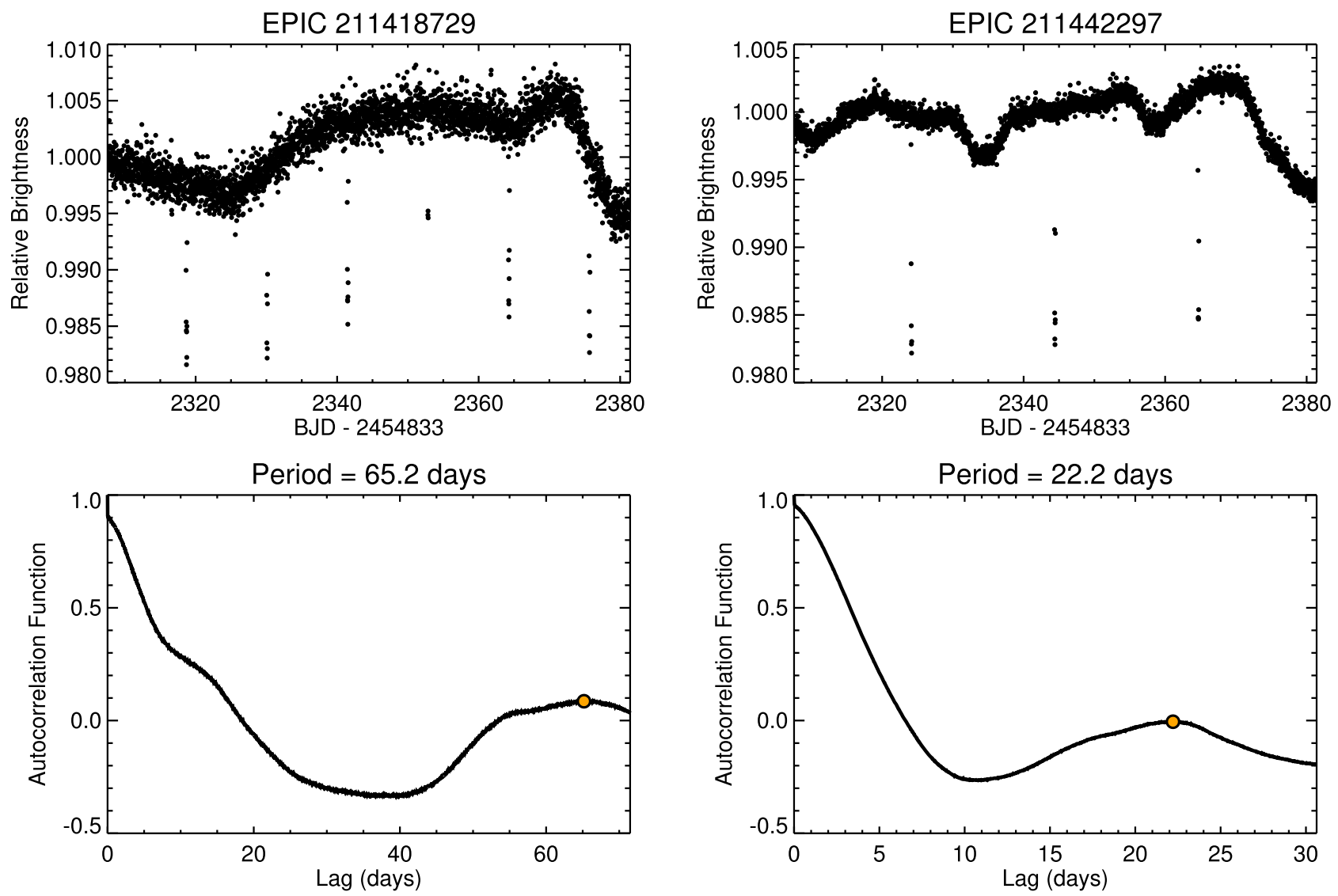

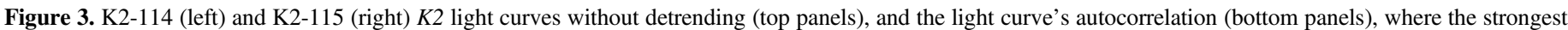

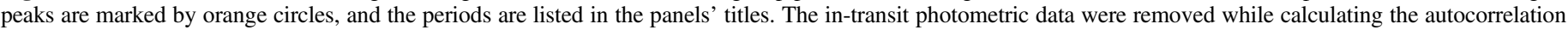

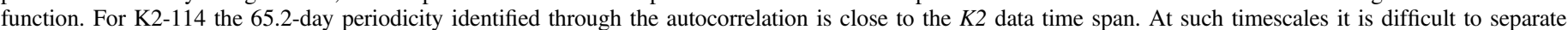

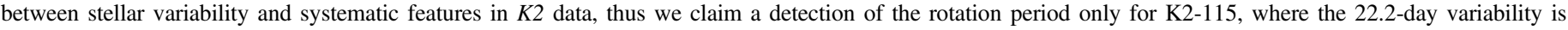
identified visually in the light curve. We estimate a $10 \%$ uncertainty on that rotation period due to differential rotation.

proximity to the $K 2$ time span means that we cannot reliably determine that that variability is stellar and not related to longterm systematic features.

\subsection{Ground-based Photometry}

While gathering the RVs of the two targets we also acquired ground-based light curves of additional transit events. The target's brightness and transit depth seen in $K 2$ data make these transits observable from the ground using $1 \mathrm{~m}$ class telescopes. Additional ground-based transits improve both the precision and accuracy of the transit ephemeris. The precision is improved due to the long time span between the transits observed by $K 2$ and those observed from the ground, which is at least several times longer than a $K 2$ campaign. The transit ephemeris accuracy is improved using additional ground-based transits, since the half-hour sampling of the $K 2$ long-cadence data can lead to a biased ephemeris in the case of an outlier measurement during one of the transits' ingress or egress (Benneke et al. 2017). This is caused by a combination of the small number of transits within a $K 2$ campaign for objects with relatively long periods as studied here, and the duration of the ingress/egress being comparable to the $K 2$ long-cadence integration time (30 minutes).

These observations were done with the LCO network of $1 \mathrm{~m}$ telescopes and the KELT follow-up network, as described below.

\subsubsection{Las Cumbres Observatory (LCO)}

LCO is a fully robotic network of telescopes deployed at six sites around the globe in both hemispheres (two more sites are planned to be added by 2018; Brown et al. 2013).

The egress of K2-114b was observed on 2016 February 17 using a $1 \mathrm{~m}$ telescope at the Cerro Tololo Inter-American Observatory (CTIO), Chile. The camera used was the Sinistro custom-built imaging camera, with back-illuminated $4 \mathrm{~K} \times 4 \mathrm{~K}$ Fairchild Imaging $\mathrm{CCD}$ with $15 \mu \mathrm{m}$ pixels (CCD486 BI). With a plate scale of 0".387/pixel, the Sinistro cameras deliver a field of view (FOV) of $26 ! 6 \times 26 ! 6$. The cameras are read out by four amplifiers with a readout time of $\approx 45 \mathrm{~s}$. We used the $i$-band filter, with an exposure time of $180 \mathrm{~s}$ and a slight defocus of the telescope $(1 \mathrm{~mm})$, to spread out the PSF over more pixels and eliminate the risk of saturation at the core of the PSF. Images were reduced by the standard LCO pipeline (Brown et al. 2013), and aperture photometry was performed in the manner set out in Penev et al. (2013) through a fully automated pipeline developed in our group (N. Espinoza et al. 2017, in preparation).

For K2-115b, LCO observed an almost complete transit on 2016 February 22 with a $1 \mathrm{~m}$ telescope at the Siding Spring Observatory (SSO), Australia, and an egress on 2016 March 13 with a $1 \mathrm{~m}$ telescope at the South African Astronomical Observatory (SAAO), South Africa. These two observations were made using the older SBIG cameras (which have since been replaced by Sinistro cameras). The SBIG cameras 

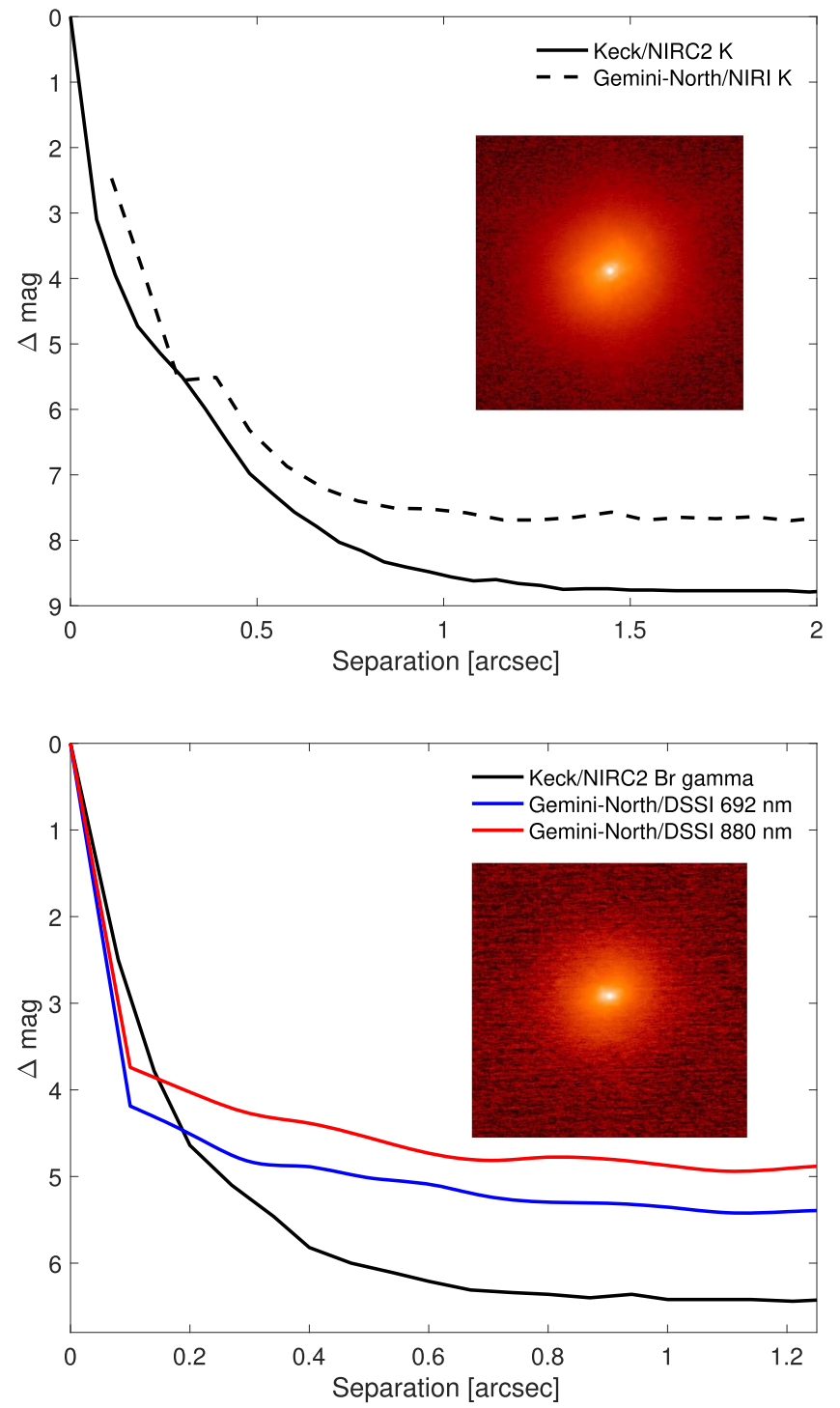

Figure 4. Top: K2-114 contrast curves obtained by Keck/NIRC2 (black solid line; $K$ filter) and Gemini-North/NIRI (dashed black line; $K$ filter). Bottom: K2-115 contrast curves, obtained by Keck/NIRC2 in the $\mathrm{Br}$ gamma filter (black solid line) and Gemini-North with DSSI (speckle imaging) in the $692 \mathrm{~nm}$ (blue solid line) and $880 \mathrm{~nm}$ (red solid line). In both panels the insets show the image obtained by Keck/NIRC2 in the $K$ band spanning $3^{\prime \prime}$ on the side.

Table 2

Spectroscopic Parameters

\begin{tabular}{lcc}
\hline \hline Parameter & K2-114 & K2-115 \\
\hline$T_{\text {eff }}(\mathrm{K})$ & $5014 \pm 60$ & $5544 \pm 60$ \\
$\log g(\mathrm{cgs})$ & $4.42 \pm 0.07$ & $4.33 \pm 0.07$ \\
{$[\mathrm{Fe} / \mathrm{H}](\mathrm{dex})$} & $0.41 \pm 0.04$ & $-0.23 \pm 0.04$ \\
$V \sin (I)\left(\mathrm{km} \mathrm{s}^{-1}\right)$ & $<2$ & $<2$ \\
$S_{H K}$ & $0.258 \pm 0.051$ & $0.172 \pm 0.014$ \\
$\log R_{H K}^{\prime}(\mathrm{dex})$ & $-4.854 \pm 0.13$ & $-4.966 \pm 0.078$ \\
\hline
\end{tabular}

featured front-illuminated $4 \mathrm{~K} \times 4 \mathrm{~K} \mathrm{KAF}-16803 \mathrm{CCDs}$ with $9 \mu \mathrm{m}$ pixels. With a plate scale of 0 ." $232 /$ pixel these cameras have a FOV of $15.8 \times 15$ !. We used $2 \times 2$ pixel binning, which results in a readout time of $15.5 \mathrm{~s}$. We again used the $i$-band, exposure times of $180 \mathrm{~s}$, and a telescope defocus of $1 \mathrm{~mm}$. Data were reduced to light curves in the same manner as
Table 3

Keck/HIRES Radial Velocities

\begin{tabular}{lrc}
\hline \hline $\begin{array}{l}\text { Time } \\
\text { BJD }\end{array}$ & $\begin{array}{c}\mathrm{RV} \\
\left(\mathrm{m} \mathrm{s}^{-1}\right)\end{array}$ & $\begin{array}{c}\mathrm{RV} \text { err } \\
\left(\mathrm{m} \mathrm{s}^{-1}\right)\end{array}$ \\
\hline K2-114 & & \\
2457422.89476 & 152.4 & 5.8 \\
2457789.92031 & -50.5 & 6.1 \\
2457802.86068 & -194.3 & 6.8 \\
2457853.77744 & 104.4 & 5.6 \\
2457886.80575 & -32.3 & 8.2 \\
\hline K2-115 & & \\
2457422.86801 & -8.9 & 6.5 \\
2457774.91483 & 37.4 & 7.3 \\
2457789.90837 & -74.0 & 7.1 \\
2457802.87129 & 91.4 & 7.1 \\
2457804.81710 & 35.1 & 7.3 \\
2457830.82364 & -58.9 & 7.8 \\
2457853.76652 & -33.7 & \\
\hline
\end{tabular}
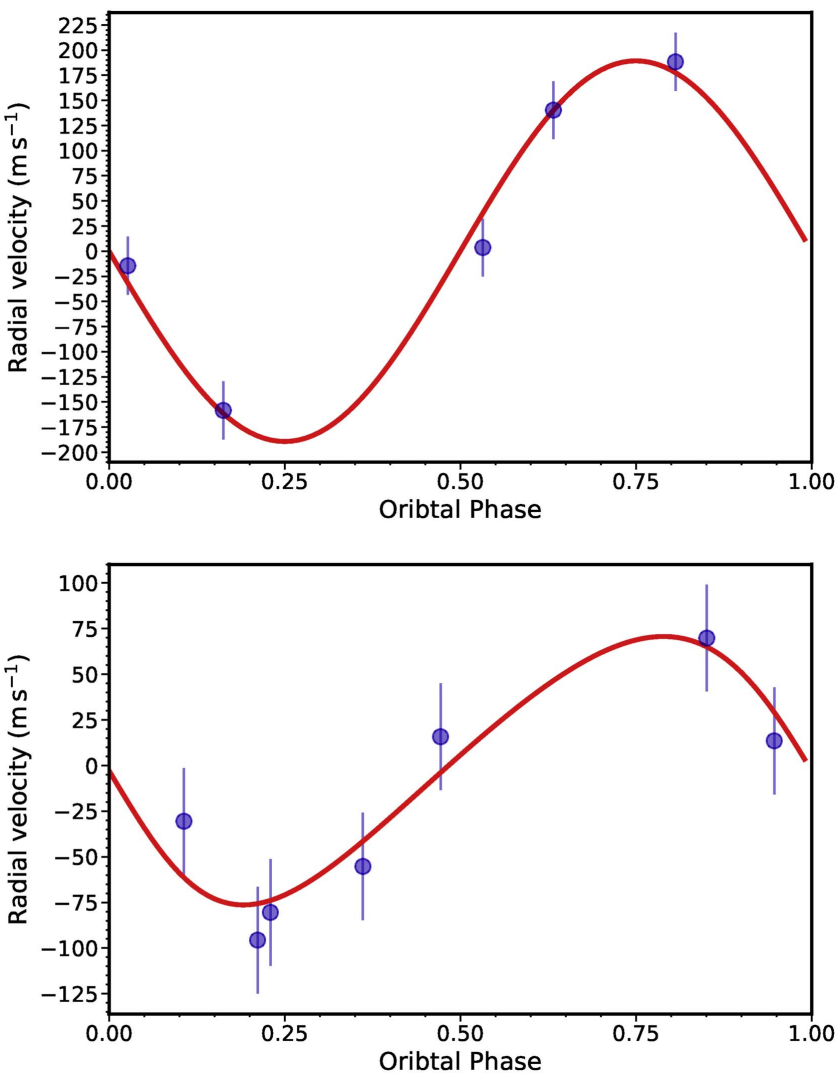

Figure 5. Phase-folded Keck/HIRES RV curves of K2-114 (top) and K2-115 (bottom). The RVs are marked with blue circles and error bars, and the fitted model is marked with a red solid line. The error bars shown in the plots include the jitter term added in quadrature (see Section 4).

set out for the Sinistro camera reduction. We note that the combination of a smaller FOV (fewer reference stars) and front-illuminated CCDs (large intra-pixel variation and lower quantum efficiency) means that the precision of the light curves derived from the SBIG cameras is much lower than that of the Sinistro cameras. Additionally, site conditions at CTIO are typically much better for photometry than those at either SAAO or SSO, which also contributes to the precision of the photometry. 
Table 4

Ground-based Photometry

\begin{tabular}{lcc}
\hline \hline Time & $\begin{array}{c}\text { Relative } \\
\text { FJD }\end{array}$ & $\begin{array}{c}\text { Relative } \\
\text { Flux Error }\end{array}$ \\
\hline K2-114-LCO & & \\
2457436.53253 & 0.9818 & 0.0036 \\
2457436.53508 & 0.9846 & 0.0035 \\
2457436.53763 & 0.9865 & 0.0036 \\
K2-115-LCO & & \\
2457440.91603 & 0.9910 & 0.0056 \\
2457440.91825 & 1.0012 & 0.0050 \\
2457440.92048 & 0.9909 & 0.0048 \\
K2-115-ULMT $0.6 \mathrm{~m}$ & & 0.0015 \\
2457805.79750 & 0.9979 & 0.0015 \\
2457805.79880 & 0.9995 & 0.0015 \\
2457805.80032 & 1.0005 & \\
\hline
\end{tabular}

(This table is available in its entirety in machine-readable form.)

The LCO light curves are shown in Figure 2 and listed in Table 4.

\subsubsection{University of Louisville Manner $0.6 \mathrm{~m}$ Telescope}

We observed one full transit of K2-115b with short segments of out-of-transit baselines using the University of Louisville Manner Telescope (ULMT) located at the Mt. Lemmon summit of Steward Observatory, AZ, on 2017 February 21 with no filter. ULMT is a member of the KELT follow-up network, composed of $1 \mathrm{~m}$ class telescopes and smaller telescopes dedicated to the photometric follow-up of transiting planet candidates. The observations employed a $0.6 \mathrm{~m} / \mathrm{s} \mathrm{RC}$ Optical Systems Ritchey-Chrétien telescope and SBIG STX$16803 \mathrm{CCD}$ with a $4 \mathrm{k} \times 4 \mathrm{k}$ array of $9 \mu \mathrm{m}$ pixels, yielding a $26 ! 6 \times 26 ! 6$ FOV and 0!'39 pixel $^{-1}$ image scale. The telescope was defocused, resulting in a "donut"-shaped stellar PSF, and guiding was applied to maintain stable pointing.

The telescope control computer malfunctioned at about the time egress started, but was recovered about 25 minutes later.

The image sequence was bias-, dark-, and flat-field corrected using AstroImageJ (AIJ; Collins et al. 2017). We also used AIJ to extract differential photometry using aperture photometry. An iterative $2 \sigma$ cleaning routine was employed to exclude outlier pixels and pixels containing flux from nearby stars from the background region. To normalize the target light curve and have the out-of-transit flux level set to unity we used a comparison ensemble of 10 stars that produced the lowest model fit residuals.

The ULMT $0.6 \mathrm{~m} \mathrm{K2-115} \mathrm{light} \mathrm{curve} \mathrm{is} \mathrm{shown} \mathrm{in} \mathrm{Figure} 2$ and listed in Table 4.

\section{Data Analysis and Results}

To derive accurate parameters for each system we performed a global modeling of the available photometric and spectroscopic observations. We used the model-fitting procedure described in Zhou et al. (2017). This includes making use of the K2 photometry, ground-based follow-up light curves, RV measurements, and spectroscopic atmospheric properties of the host stars. The light curves are modeled as per Mandel \& Agol (2002), where the free parameters are the orbital period $P$, mid transit time $T_{0}$, the planet-to-star-radii ratio $R_{p} / R_{s}$, normalized orbital semimajor axis distance $a / R_{s}$, line-of-sight orbital inclination $i$, and orbital eccentricity parameters $\sqrt{e} \cos \omega$ and $\sqrt{e} \sin \omega$, where $e$ is the eccentricity and $\omega$ the argument of periastron. The 30-minute duration of the $K 2$ long-cadence exposures is accounted for by integrating over 10 model steps per exposure. Quadratic limb-darkening coefficients are interpolated from Claret (2004) to the atmospheric parameters of each star, and held fixed during the fitting. For the ULMT observation of K2-115 (Section 3.4.2), obtained without a filter, we adopted the same limb-darkening parameters as that of the $K 2$ light curves.

The RVs are modeled by a Keplerian orbit, with additional free parameters for the orbit RV semi-amplitude $K$ and systemic center-of-mass RV $\gamma$. We include a fitted jitter term $s$ to model the RVs per Haywood et al. (2016). Since the transit duration is dictated by the stellar density, we also make use of the precise $K 2$ photometry to refine the stellar parameters. We interpolate the Dartmouth stellar isochrones (Dotter et al. 2008) over the axes of stellar atmospheric effective temperature $T_{\text {eff }}$, mean stellar density $\rho_{s}$, and metallicity $[\mathrm{Fe} / \mathrm{H}]$, to derive a surface gravity $\log g$.

The transit-derived stellar density and spectroscopically constrained effective temperatures are plotted in Figure 6 against solar metallicity isochrones to illustrate this isochrone interpolation process. We reject solutions that yield system ages older than $13 \mathrm{Gyr}$, the age of the thin disk of the galaxy (Knox et al. 1999). At each iteration, we include a loglikelihood term calculated between the transit-derived $\log g$ with that measured from spectroscopy. The posterior probability distribution is explored via a Markov Chain Monte Carlo (MCMC) analysis, using the affine invariant sampler emcee (Foreman-Mackey et al. 2013). Gaussian priors are applied for the stellar atmospheric parameters $T_{\text {eff }}$ and $[\mathrm{Fe} / \mathrm{H}]$, and all other parameters are assumed to follow uniform priors.

For both objects we ran two fits, one that assumes the orbit is circular and another that fits for the eccentricity $e$ and argument of periastron $\omega$. For K2-114b, the eccentric orbit fit did not give a statistically significant eccentricity, hence we adopt the circular orbit model. The upper limits on the orbital eccentricity are 0.06 and 0.41 at $1 \sigma$ and $3 \sigma$, respectively.

For K2-115, the eccentricity is measured at a statistical significance of close to $2 \sigma$, hence we adopted the eccentric orbit model. We tested the significance of the measured orbital eccentricity by refitting this system using a Beta function prior distribution on the eccentricity following Kipping (2014). That analysis gave consistent results at the $1 \sigma$ level. Another reason for adopting the eccentric orbit model was that it gave consistent results for the derived stellar parameters (mass, radius, and age) with those derived when fitting using only stellar isochrones (with priors on the spectral parameters $T_{\text {eff }}$, $\log g$, and $[\mathrm{Fe} / \mathrm{H}]$ ) and without the light curve and RV curve. The derived stellar parameters from the circular orbit model fit are not consistent with the results from fitting using stellar evolutionary models alone.

The $68 \%$ confidence region for the model fit free parameters, as well as a series of inferred system parameters, are listed in Table 5. The best-fit transit light curve models are shown in Figure 2 and the RV fits are shown in Figure 5.

\section{Discussion and Conclusions}

Both $\mathrm{K} 2-114 \mathrm{~b}$ and $\mathrm{K} 2-115 \mathrm{~b}$ are among the longest-period transiting gas giant planets with a measured mass. In fact, according to the NASA Exoplanet Archive (Akeson 

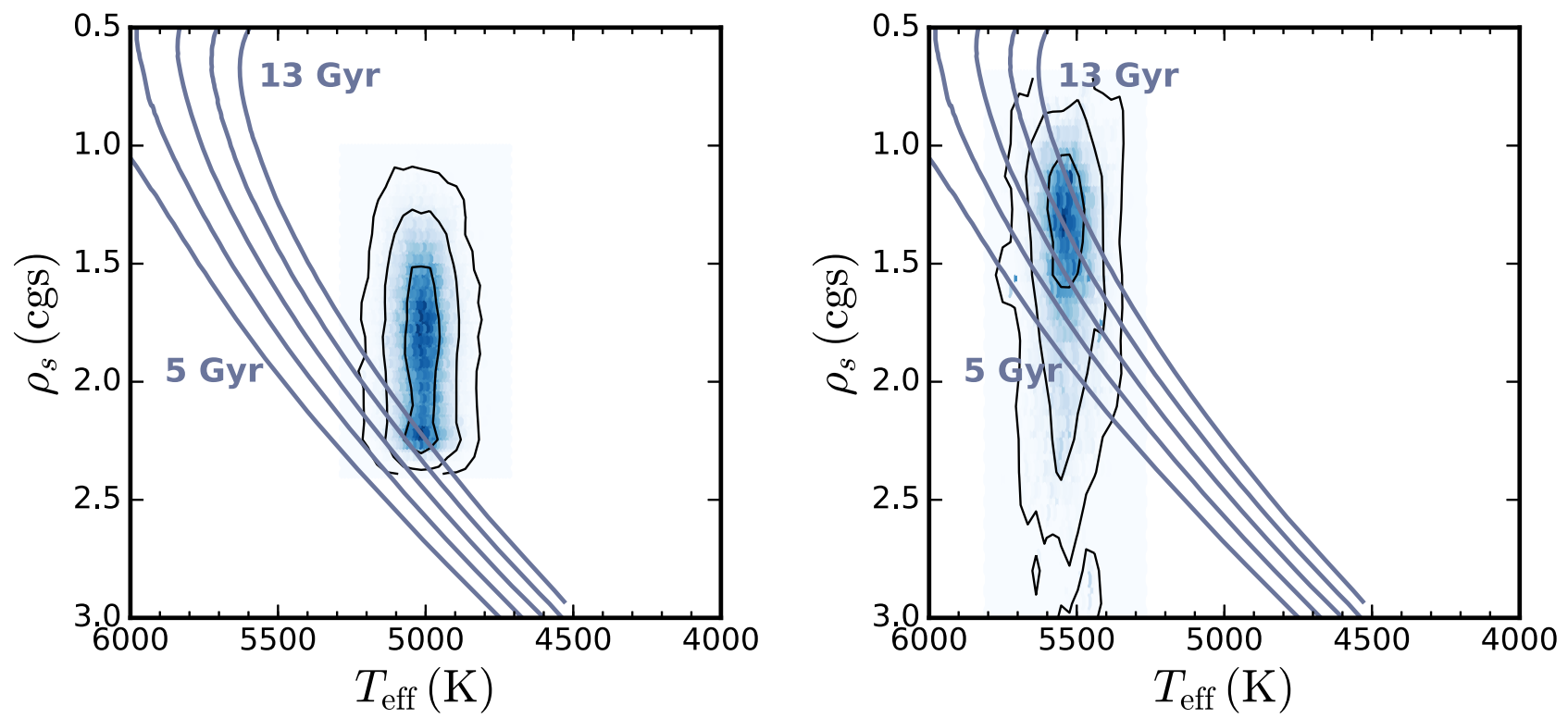

Figure 6. Stellar parameters are derived via interpolation of the Dartmouth isochrones. The stellar density $\left(\rho_{s}\right)$, derived from the transit light curve, and the spectroscopic effective temperature $T_{\text {eff }}$ and metallicity $[\mathrm{Fe} / \mathrm{H}]$ are compared against isochrone tracks at each MCMC iteration to constrain the stellar properties. The stellar densities and effective temperatures of K2-114 (left) and K2-115 (right) are plotted. The Dartmouth solar metallicity isochrones at ages of 5, 7, 9, 11, and 13 Gyrs are drawn as a guide. Note that solutions yielding ages $>13 \mathrm{Gyr}$ are removed during the derivation of system and stellar parameters in the global analysis, as that age is older than the age of the thin disk of the galaxy.

et al. 2013), K2-115b is currently ${ }^{27}$ the longest-period $K 2$ transiting exoplanet with a well constrained mass (but see Bayliss et al. 2017).

The number of RVs we have accumulated for each system is relatively small, with five for K2-114 and seven for K2-115. The relatively small number of RVs results in a relatively poor constraint of the orbital eccentricity and RV semi-amplitude. The latter has an uncertainty of $12 \%$ for $\mathrm{K} 2-114$ and close to $22 \%$ for K2-115, leading to similar uncertainties on the two planet masses.

Note that both host stars are relatively old, with ages close to $10 \mathrm{Gyr}$, although with the typical large age uncertainties (see Table 5). For K2-115b, despite the host star old age, the combination of the measured orbital eccentricity $\left(e=0.137_{-0.074}^{+0.072}\right)$ and orbital separation $\left(a / R_{s}=33.8_{-1.7}^{+2.3}\right)$ suggests that if the orbit is indeed eccentric, that eccentricity is primordial, since it is not expected to be tidally circularized within the host star's lifetime (e.g., Mazeh 2008).

Figure 7 top panel shows the planet mass-radius diagram for gas giant plants, with $R_{p}>0.6 R_{\mathrm{J}}$, and with well-measured planet radius and mass. Those include 273 planets listed on the NASA Exoplanet Archive with planet radius errors smaller than $0.15 R_{\mathrm{J}}$ and planet mass errors below $20 \%$ of the planet mass itself. Not included in that sample are circumbinary planets and directly imaged planets. The black and gray solid lines show the range of theoretical planet radii where the planet radius grows as the mass of its rocky core decreases (Fortney et al. 2007). The dashed gray lines are equal mean density lines. The two new planets, K2-114b and K2-115b, are marked in red. Both planets are not inflated compared to theoretical expectations, unlike many other planets in the diagram. Their positions are close to or consistent with theoretical expectations for a planet with little to no rocky core, for K2-115b, and a planet with a significant rocky core for K2-114b.

27 As of 2017 June 1st.
The difference in the expected core mass between the two planets, combined with the larger planet mass of K2-114b compared to $\mathrm{K} 2-115 \mathrm{~b}$, agrees with the empirical correlation between heavy element mass and planet mass for gas giants (Miller \& Fortney 2011; Thorngren et al. 2016). The differences in the host star metallicity, with K2-114 being super-solar and K2-115 being sub-solar, also agrees with the gas giant planet mass-host star metallicity correlation (Miller \& Fortney 2011; Thorngren et al. 2016). These correlations allow us to estimate the planets' compositions (Espinoza et al. 2017).

The bottom panel of Figure 7 shows the planet radius-stellar irradiation diagram $\left(R_{p}-f\right)$, including the same sample of planets as in the top panel, and where the two new planets are marked in red. Their positions are consistent with the hypothesis that the $R_{p}-f$ correlation does not continue below irradiation of $10^{8} \mathrm{erg} \mathrm{s}^{-1} \mathrm{~cm}^{-2}$, where the correlation levels off and stellar irradiation does not significantly affect the planet radius. If true, this can be used as a clue for identifying the physical mechanism inflating gas giant planets, and it makes warm Jupiters good targets for testing theoretical mass-radius relations, as their radii are not affected by a physical mechanism that is currently not completely understood. However, an accurate characterization of the behavior of planet radius at low stellar irradiation requires the detection of many more warm Jupiters.

Finally, we note that the two new planets reported here are planned to be observed by $K 2$ again during Campaign 18, from 2018 May to August, when $K 2$ will re-observe the Campaign 5 field. ${ }^{28}$ If successful, this will give a 3 -year time span and therefore allow us to refine the transit ephemerides and the planet-to-star-radii ratio, looking for transit timing variations and searching for other transiting planets in those systems.

${ }^{28}$ See list of $K 2$ fields here: https://keplerscience.arc.nasa.gov/k2-fields.html. 
Table 5

Fitted and Derived Parameters

\begin{tabular}{|c|c|c|c|c|c|c|}
\hline \multirow{2}{*}{ Parameter } & \multicolumn{3}{|c|}{$\mathrm{K} 2-114$} & \multicolumn{3}{|c|}{$\mathrm{K} 2-115$} \\
\hline & Value & $+1 \sigma$ & $-1 \sigma$ & Value & $+1 \sigma$ & $-1 \sigma$ \\
\hline \multicolumn{7}{|c|}{ Fitted Parameters } \\
\hline$P$ (day) & 11.39109 & 0.00018 & 0.00017 & 20.273034 & 0.000036 & 0.000037 \\
\hline$T_{0}(\mathrm{BJD})$ & 2457174.49729 & 0.00033 & 0.00033 & 2457157.15701 & 0.00025 & 0.00025 \\
\hline$\gamma\left(\mathrm{m} \mathrm{s}^{-1}\right)$ & -36 & 14 & 14 & 22 & 15 & 12 \\
\hline$K\left(\mathrm{~m} \mathrm{~s}^{-1}\right)$ & 189 & 21 & 22 & 77 & 17 & 16 \\
\hline$\sqrt{e} \cos (\omega)$ & $0^{\mathrm{a}}$ & $\ldots$ & $\ldots$ & -0.12 & 0.25 & 0.18 \\
\hline$\sqrt{e} \sin (\omega)$ & $0^{\mathrm{a}}$ & $\ldots$ & $\cdots$ & 0.28 & 0.11 & 0.17 \\
\hline Jitter $s\left(\mathrm{~m} \mathrm{~s}^{-1}\right)$ & 29 & 12 & 25 & 24 & 16 & 8 \\
\hline$a / R_{s}$ & 24.44 & 0.42 & 0.63 & 33.8 & 2.3 & 1.7 \\
\hline$R_{p} / R_{s}$ & 0.11432 & 0.00102 & 0.00073 & 0.1254 & 0.0011 & 0.0011 \\
\hline$i(\mathrm{deg})$ & 89.53 & 0.30 & 0.25 & 88.82 & 0.15 & 0.15 \\
\hline$T_{\text {eff }}(\mathrm{K})$ & 5027 & 62 & 57 & 5560 & 56 & 58 \\
\hline$[\mathrm{Fe} / \mathrm{H}]$ & +0.410 & 0.037 & 0.035 & -0.220 & 0.035 & 0.036 \\
\hline$u_{1} K 2^{\mathrm{a}}$ & 0.5815 & $\ldots$ & $\ldots$ & 0.4430 & $\ldots$ & $\ldots$ \\
\hline$u_{2} K 2^{\mathrm{a}}$ & 0.1392 & $\cdots$ & $\cdots$ & 0.2312 & $\cdots$ & $\cdots$ \\
\hline$u_{1} \mathrm{ULMT}^{\mathrm{a}}$ & $\ldots$ & $\ldots$ & $\ldots$ & 0.4430 & $\ldots$ & $\ldots$ \\
\hline$u_{2} \mathrm{ULMT}^{\mathrm{a}}$ & $\ldots$ & $\ldots$ & $\ldots$ & 0.2312 & $\ldots$ & $\ldots$ \\
\hline$u_{1} i^{\prime \mathrm{a}}$ & 0.4225 & $\ldots$ & $\ldots$ & 0.3118 & $\ldots$ & $\ldots$ \\
\hline$u_{2} i^{\prime \mathrm{a}}$ & 0.2472 & $\cdots$ & $\cdots$ & 0.3047 & $\cdots$ & $\cdots$ \\
\hline \multicolumn{7}{|c|}{ Derived Parameters } \\
\hline$M_{s}\left(M_{\odot}\right)$ & 0.832 & 0.021 & 0.018 & 0.831 & 0.023 & 0.019 \\
\hline$R_{s}\left(R_{\odot}\right)$ & 0.828 & 0.026 & 0.022 & 0.881 & 0.049 & 0.050 \\
\hline$\rho_{s}(\mathrm{cgs})$ & 1.84 & 0.32 & 0.29 & 1.43 & 0.44 & 0.28 \\
\hline $\log g(\mathrm{cgs})$ & 4.481 & 0.044 & 0.051 & 4.461 & 0.057 & 0.041 \\
\hline Age (Gyr) & 9.9 & 2.3 & 3.2 & 10.7 & 1.7 & 4.2 \\
\hline$R_{p}\left(R_{\mathrm{J}}\right)$ & 0.942 & 0.032 & 0.020 & 1.115 & 0.057 & 0.061 \\
\hline$M_{p}\left(M_{\mathrm{J}}\right)$ & 1.85 & 0.23 & 0.22 & 0.84 & 0.18 & 0.20 \\
\hline$\rho_{p}(\mathrm{cgs})$ & 2.99 & 0.46 & 0.45 & 0.82 & 0.30 & 0.24 \\
\hline$a(\mathrm{au})$ & 0.09309 & 0.00066 & 0.00059 & 0.1367 & 0.0012 & 0.0010 \\
\hline$T_{\text {eq }}(\mathrm{K})^{\mathrm{b}}$ & 719 & 15 & 11 & 682 & 22 & 24 \\
\hline$b$ & 0.20 & 0.10 & 0.12 & 0.702 & 0.047 & 0.053 \\
\hline$T_{12}$ (day) & 0.01732 & 0.00109 & 0.0068 & 0.0343 & 0.0050 & 0.0046 \\
\hline$T_{14}$ (day) & 0.1627 & 0.0011 & 0.0010 & 0.1679 & 0.0027 & 0.0046 \\
\hline$e$ & $0^{\mathrm{a}}$ & $\ldots$ & $\ldots$ & 0.137 & 0.072 & 0.074 \\
\hline$\omega(\operatorname{deg})$ & $\ldots$ & $\ldots$ & $\ldots$ & 104 & 41 & 52 \\
\hline Distance (pc) & 481 & 20 & 15 & 417 & 26 & 25 \\
\hline$A_{V}(\mathrm{mag})$ & 0.109 & 0.072 & 0.072 & $<0.12^{\mathrm{c}}$ & $\cdots$ & $\cdots$ \\
\hline
\end{tabular}

Notes.

a Parameter was fixed during the model-fitting process.

${ }^{\mathrm{b}}$ Assuming zero albedo and no redistribution of heat.

c $3 \sigma$ upper limit given for reddening.

We are grateful to the anonymous referee for the meticulous reading of the manuscript and for providing detailed comments that helped improve this work. We are grateful to Josh Pepper for his help in coordinating our ground-based follow-up observations with the KELT follow-up network. B.J.F. acknowledges that this material is based upon work supported by the National Science Foundation Graduate Research Fellowship under grant No. 2014184874. A.V. is supported by the NSF Graduate Research Fellowship, grant No. DGE 1144152. D.D. acknowledges support provided by NASA through Hubble Fellowship grant HST-HF2-51372.001-A awarded by the Space Telescope Science Institute, which is operated by the Association of Universities for Research in Astronomy, Inc., for NASA, under contract NAS5-26555. A.C.C. acknowledges support from STFC consolidated grant number ST/M001296/1. This paper includes data collected by the $K 2$ mission. Funding for the $K 2$ mission is provided by the NASA Science Mission directorate. This work makes use of observations from the LCO network. Some of the data presented herein were obtained at the W.M. Keck Observatory, which is operated as a scientific partnership among the California Institute of Technology, the University of California, and the National Aeronautics and Space Administration. The Observatory was made possible by the generous financial support of the W.M. Keck Foundation. The authors wish to recognize and acknowledge the very significant cultural role and reverence that the summit of Maunakea has always had within the indigenous Hawaiian community. We are most fortunate to have the opportunity to conduct observations from this mountain.

Facilities: Gemini:North (DSSI, NIRI), K2, Keck:I (HIRES), Keck:II (NIRC2), LCO (SBIG, Sinistro), Euler $1.2 \mathrm{~m}$ (CORALIE). 

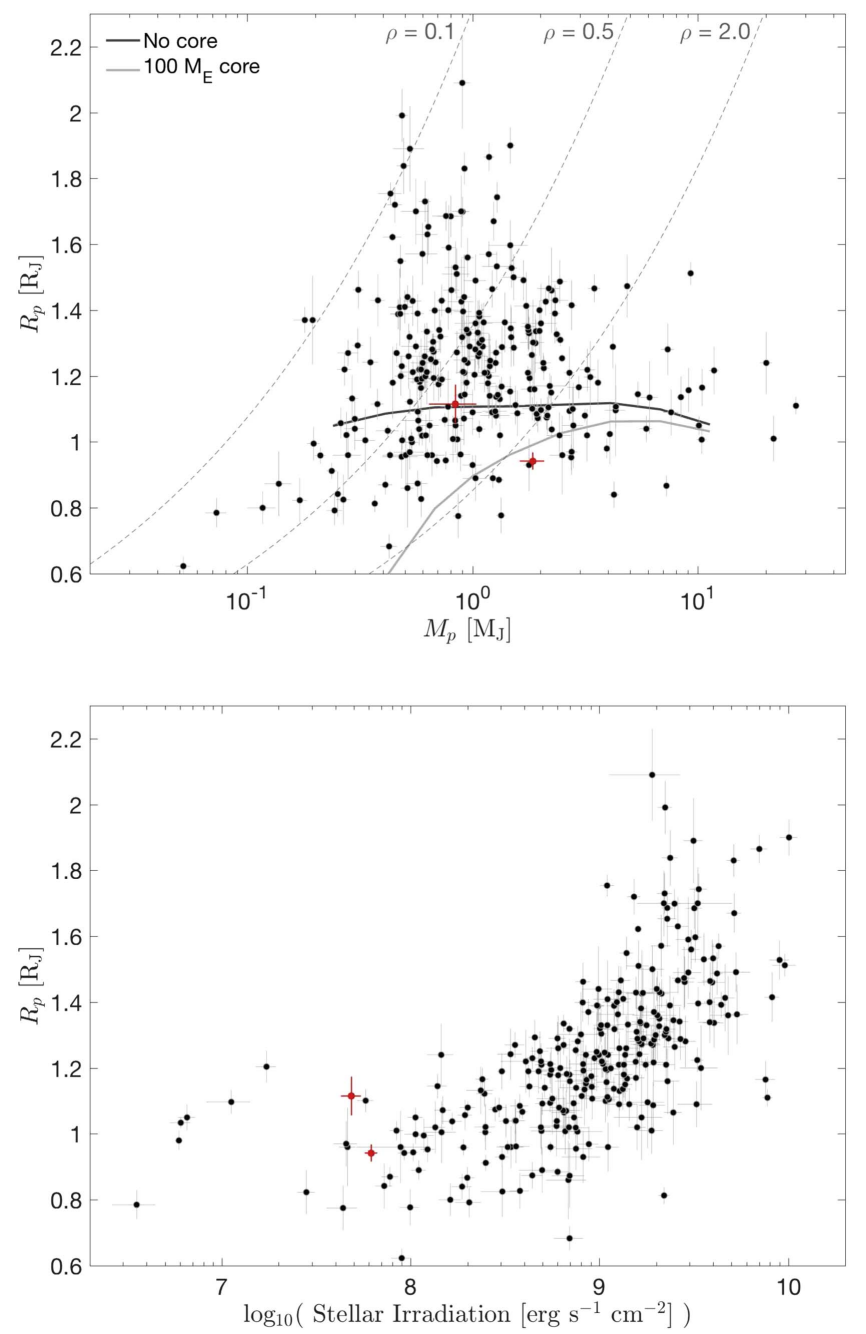

Figure 7. This figure shows the planet radius (linear scale) as a function of planet mass (log scale; top panel) and stellar irradiation (log scale; bottom panel). K2-114b and K2-115b are marked in red. In the top panel the solid lines encompass the theoretically expected region where planets are expected to reside in this parameter space (Fortney et al. 2007), between a coreless planet (solid black line) and a planet with a massive core of $100 M_{\oplus}$ (solid gray line). Both theoretical curves are for an assumed orbital star-planet separation of $0.045 \mathrm{au}$, although the planet radius changes by up to only $\approx 10 \%$ between a separation of 0.02 au and 0.10 au (Fortney et al. 2007). The dashed gray lines mark lines of equal mean density, and the density is labeled in the top part of the panel, in units of $\mathrm{g} \mathrm{cm}^{-3}$. Both panels show in black 273 planets with $R_{p}$ over $0.6 R_{\mathrm{J}}$, a planet radius error below $0.15 R_{\mathrm{J}}$, and a planet mass error below $20 \%$ of the planet mass itself, to include only planets with well-measured masses and radii. Circumbinary planets and directly imaged planets are excluded from these plots. Data shown in these plots were taken from the NASA Exoplanet Archive (Akeson et al. 2013) on 2017 June 1.

\section{Appendix \\ K2 Warm Jupiter Transit Candidate Identified as a False Positive}

In addition to the two $K 2$ transiting warm Jupiters whose confirmations as planets were described above, we have identified one $K 2$ transiting warm Jupiter candidate, EPIC 212504617 ( $P=39.26$ days), as a stellar binary, meaning it is a false positive. The $K 2$ Campaign 6 phase-folded transit light curve is shown in Figure 8, derived in the same way as described in Section 3.1.

We have identified EPIC 212504617 as a stellar binary using two RVs obtained with the CORALIE spectrograph, mounted on

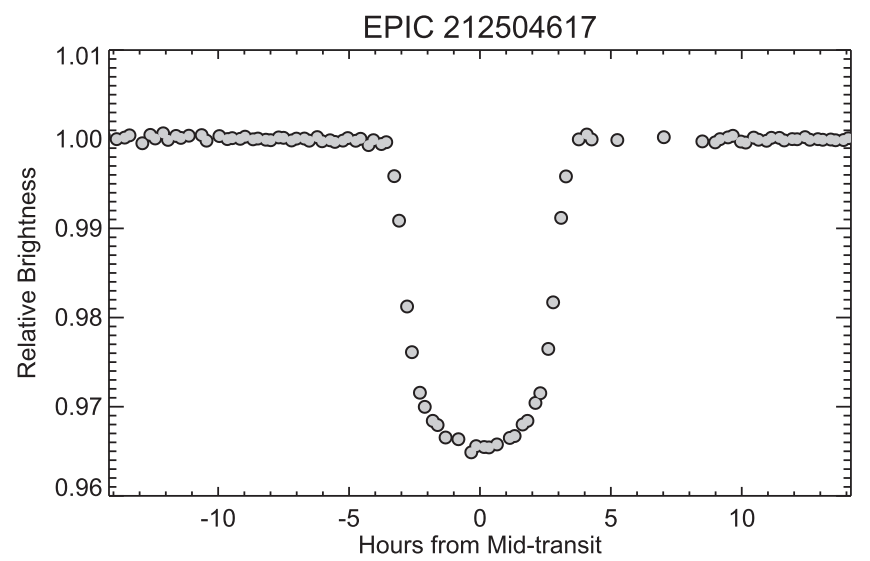

Figure 8. K2 Campaign 6 phase-folded transit light curve of EPIC 212504617, a warm Jupiter transiting candidate identified as a false positive.

Table 6

EPIC 212504617 Euler/CORALIE Radial Velocities

\begin{tabular}{lcc}
\hline \hline $\begin{array}{l}\text { Time } \\
\text { BJD }\end{array}$ & $\begin{array}{c}\mathrm{RV} \\
\left(\mathrm{km} \mathrm{s}^{-1}\right)\end{array}$ & $\begin{array}{c}\mathrm{RV} \text { err } \\
\left(\mathrm{km} \mathrm{s}^{-1}\right)\end{array}$ \\
\hline 2457493.76167 & -24.667 & 0.043 \\
2457561.53544 & -40.603 & 0.070 \\
\hline
\end{tabular}

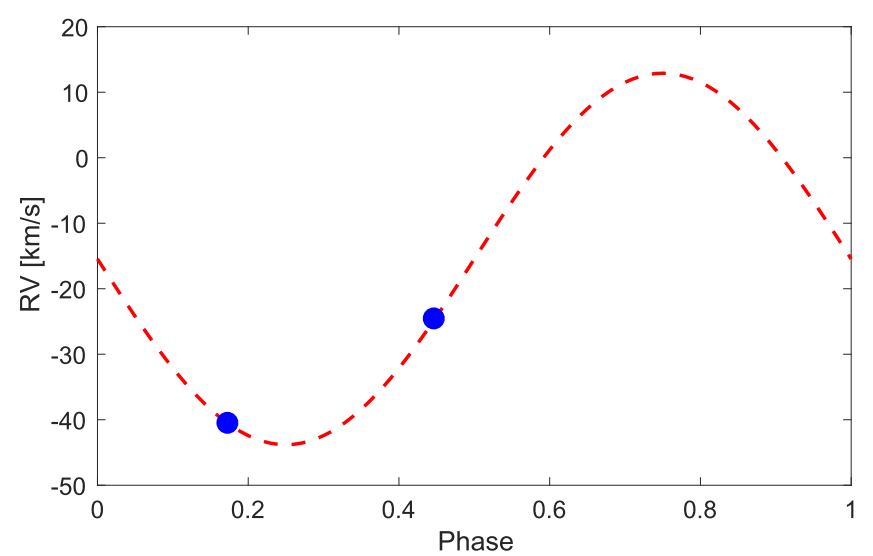

Figure 9. Euler/CORALIE RVs of EPIC 212504617 phase-folded at the transit period of $P=39.26$ day. The RVs are marked in blue (error bars are smaller than the marker size), and the dashed line shows the best-fit $e=0$ orbital solution. Phase zero is the primary eclipse (transit) phase. The fitted semi-amplitude is $28.4 \mathrm{~km} \mathrm{~s}^{-1}$. Since this is a two-parameter model fitted to two RVs, this model is used only as an estimate for the orbital RV variation. This estimate rules out the possibility of a substellar mass companion, as discussed in more detail in the text.

the Euler $1.2 \mathrm{~m}$ telescope in La Silla, Chile. CORALIE is a highresolution $(R=60,000)$ fiber-fed echelle spectrograph that covers the wavelength range from 3900 to $6800 \AA$ (Queloz et al. 2001). Observations were made with a simultaneous Fabry-Pérot fiber to provide accurate wavelength calibration, and reduced via the standard CORALIE pipeline. The stellar spectra were cross-correlated against a numerical mask with nonzero zones corresponding to stellar absorption features at zero velocity.

Using CORALIE two observations of the candidate EPIC 212504617 were made, each with an exposure time of $2700 \mathrm{~s}$. The two RVs have a difference of $16 \mathrm{~km} \mathrm{~s}^{-1}$. Those RVs are listed in Table 6 and shown in Figure 9. The best-fit circular orbit model for these RVs gives a semi-amplitude of $K=28.4 \mathrm{~km} \mathrm{~s}^{-1}$ and a systemic velocity of $\gamma=-15.5 \mathrm{~km} \mathrm{~s}^{-1}$. 
This is a two-parameter model fitted to only two RVs, hence this model is used only as an estimate for the orbital RV variation. Given the transit period of $P=39.26$ days and the host star's estimated mass of $1.01 M_{\odot}$ (Huber et al. 2016), the circular orbit RV semi-amplitude predicts a companion mass of about $0.6 M_{\odot}$. Even when invoking a high eccentricity of 0.95 the companion mass should be at least $45 M_{\mathrm{J}}$ for the system to show an RV variability of $16 \mathrm{~km} \mathrm{~s}^{-1}$. Therefore, the companion cannot be a planet and is highly unlikely to be substellar.

\section{ORCID iDs}

Avi Shporer (iD https://orcid.org/0000-0002-1836-3120 George Zhou (ib https://orcid.org/0000-0002-4891-3517 Benjamin J. Fulton (iD https://orcid.org/0000-0003-3504-5316 Andrew Vanderburg (iD https://orcid.org/0000-0001-7246-5438 Nestor Espinoza (iD https://orcid.org/0000-0001-9513-1449

Karen Collins (iD https://orcid.org/0000-0001-6588-9574

Daniel Bayliss (D) https://orcid.org/0000-0001-6023-1335

William D. Cochran (iD https://orcid.org/0000-0001-9662-3496

Knicole Colón (10 https://orcid.org/0000-0001-8020-7121

Diana Dragomir (iD https://orcid.org/0000-0003-2313-467X

Andrew W. Howard (i) https://orcid.org/0000-0001-8638-0320

Steve B. Howell (iD https://orcid.org/0000-0002-2532-2853

Howard Isaacson (iD https://orcid.org/0000-0002-0531-1073

Evan Sinukoff (ib https://orcid.org/0000-0002-5658-0601

Robert Siverd (ib https://orcid.org/0000-0001-5016-3359

\section{References}

Akeson, R. L., Chen, X., Ciardi, D., et al. 2013, PASP, 125, 989

Alibert, Y., Mordasini, C., Benz, W., \& Winisdoerffer, C. 2005, A\&A, 434, 343

Arras, P., \& Socrates, A. 2010, ApJ, 714, 1

Baraffe, I., Chabrier, G., Fortney, J., \& Sotin, C. 2014, in Protostars and Planets VI, ed. H. Beuther et al. (Tucson, AZ: Univ. Arizona Press), 763

Batygin, K., \& Stevenson, D. J. 2010, ApJL, 714, L238

Bayliss, D., Hojjatpanah, S., Santerne, A., et al. 2017, AJ, 153, 15

Benneke, B., Werner, M., Petigura, E., et al. 2017, ApJ, 834, 187

Bodenheimer, P., Lin, D. N. C., \& Mardling, R. A. 2001, ApJ, 548, 466

Brown, T. M., Baliber, N., Bianco, F. B., et al. 2013, PASP, 125, 1031

Burrows, A., Hubeny, I., Budaj, J., \& Hubbard, W. B. 2007, ApJ, 661, 502

Butler, R. P., \& Marcy, G. W. 1996, ApJL, 464, L153

Ciardi, D. R., Beichman, C. A., Horch, E. P., \& Howell, S. B. 2015, ApJ, 805,16

Claret, A. 2004, A\&A, 428, 1001

Collins, K. A., Kielkopf, J. F., Stassun, K. G., \& Hessman, F. V. 2017, AJ, 153,77
Dong, S., Katz, B., \& Socrates, A. 2014, ApJL, 781, L5

Dotter, A., Chaboyer, B., Jevremović, D., et al. 2008, ApJS, 178, 89

Enoch, B., Collier Cameron, A., \& Horne, K. 2012, A\&A, 540, A99

Espinoza, N., Fortney, J. J., Miguel, Y., Thorngren, D., \& Murray-Clay, R. 2017, ApJL, 838, L9

Fabrycky, D., \& Tremaine, S. 2007, ApJ, 669, 1298

Foreman-Mackey, D., Hogg, D. W., Lang, D., \& Goodman, J. 2013, PASP, 125,306

Fortney, J. J., Marley, M. S., \& Barnes, J. W. 2007, ApJ, 659, 1661

Fulton, B. J., Collins, K. A., Gaudi, B. S., et al. 2015, ApJ, 810, 30

Furlan, E., Ciardi, D. R., Everett, M. E., et al. 2017, AJ, 153, 71

Ginzburg, S., \& Sari, R. 2016, ApJ, 819, 116

Haywood, R. D., Collier Cameron, A., Unruh, Y. C., et al. 2016, MNRAS, 457, 3637

Hodapp, K. W., Jensen, J. B., Irwin, E. M., et al. 2003, PASP, 115, 1388

Horch, E. P., Gomez, S. C., Sherry, W. H., et al. 2011, AJ, 141, 45

Horch, E. P., Howell, S. B., Everett, M. E., \& Ciardi, D. R. 2012, AJ, 144, 165

Horch, E. P., Veillette, D. R., Baena Gallé, R., et al. 2009, AJ, 137, 5057

Howell, S. B., Sobeck, C., Haas, M., et al. 2014, PASP, 126, 398

Huang, C., Wu, Y., \& Triaud, A. H. M. J. 2016, ApJ, 825, 98

Huber, D., Bryson, S. T., Haas, M. R., et al. 2016, ApJS, 224, 2

Isaacson, H., \& Fischer, D. 2010, ApJ, 725, 875

Kipping, D. M. 2014, MNRAS, 444, 2263

Knox, R. A., Hawkins, M. R. S., \& Hambly, N. C. 1999, MNRAS, 306, 736

Kolbl, R., Marcy, G. W., Isaacson, H., \& Howard, A. W. 2015, AJ, 149, 18

Komacek, T. D., \& Youdin, A. N. 2017, ApJ, 844, 94

Kovács, G., Zucker, S., \& Mazeh, T. 2002, A\&A, 391, 369

Laughlin, G., Crismani, M., \& Adams, F. C. 2011, ApJL, 729, L7

Lin, D. N. C., Bodenheimer, P., \& Richardson, D. C. 1996, Natur, 380, 606

Lopez, E. D., \& Fortney, J. J. 2016, ApJ, 818, 4

Mandel, K., \& Agol, E. 2002, ApJL, 580, L171

Mazeh, T. 2008, in EAS Publications Ser. 29, Tidal Effect in Stars, Planets and Disks, ed. M.-J. Goupil \& J.-P. Zahn (Les Ulis: EDP Sciences), 1

McQuillan, A., Mazeh, T., \& Aigrain, S. 2014, ApJS, 211, 24

Miller, N., \& Fortney, J. J. 2011, ApJL, 736, L29

Penev, K., Bakos, G. Á., Bayliss, D., et al. 2013, AJ, 145, 5

Petigura, E. A. 2015, PhD thesis, Univ. California, Berkeley

Queloz, D., Mayor, M., Udry, S., et al. 2001, Msngr, 105, 1

Rasio, F. A., \& Ford, E. B. 1996, Sci, 274, 954

Reinhold, T., Reiners, A., \& Basri, G. 2013, A\&A, 560, A4

Ricker, G. R., Winn, J. N., Vanderspek, R., et al. 2015, JATIS, 1, 014003

Showman, A. P., \& Guillot, T. 2002, A\&A, 385, 166

Steffen, J. H., Ragozzine, D., Fabrycky, D. C., et al. 2012, PNAS, 109, 7982

Sullivan, P. W., Winn, J. N., Berta-Thompson, Z. K., et al. 2015, ApJ, 809, 77

Thorngren, D. P., Fortney, J. J., Murray-Clay, R. A., \& Lopez, E. D. 2016, ApJ, 831, 64

Tremblin, P., Chabrier, G., Mayne, N. J., et al. 2017, ApJ, 841, 30

Vanderburg, A., \& Johnson, J. A. 2014, PASP, 126, 948

Vanderburg, A., Latham, D. W., Buchhave, L. A., et al. 2016, ApJS, 222, 14

Vogt, S. S., Allen, S. L., Bigelow, B. C., et al. 1994, Proc. SPIE, 2198, 362

Weiss, L. M., Marcy, G. W., Rowe, J. F., et al. 2013, ApJ, 768, 14

Youdin, A. N., \& Mitchell, J. L. 2010, ApJ, 721, 1113

Zhou, G., Bakos, G. Á., Hartman, J. D., et al. 2017, AJ, 153, 211 